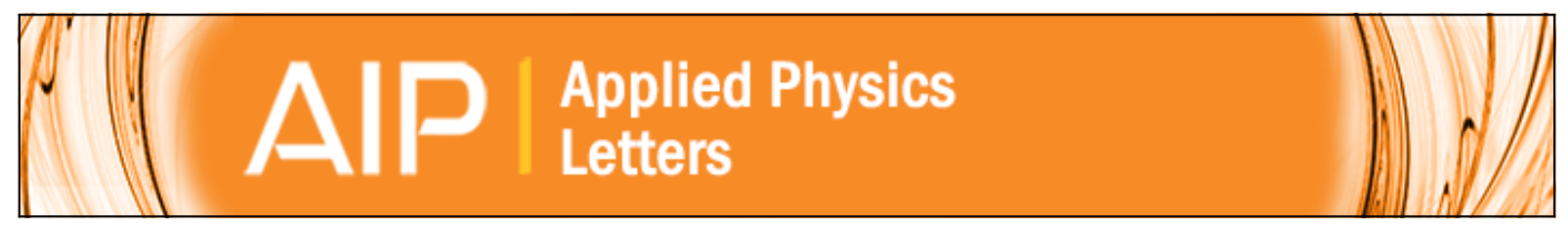

\title{
Holographic fabrication of three-dimensional orthorhombic and tetragonal photonic crystal templates using a diffractive optical element
}

Zsolt Poole, Di Xu, Kevin P. Chen, Isai Olvera, Kris Ohlinger, and Yuankun Lin

Citation: Applied Physics Letters 91, 251101 (2007); doi: 10.1063/1.2822844

View online: http://dx.doi.org/10.1063/1.2822844

View Table of Contents: http://scitation.aip.org/content/aip/journal/apl/91/25?ver=pdfcov

Published by the AIP Publishing

\section{Articles you may be interested in}

Phase tunable holographic fabrication for three-dimensional photonic crystal templates by using a single optical element

Appl. Phys. Lett. 94, 231116 (2009); 10.1063/1.3149705

Holographic fabrication of diamondlike photonic crystal template using two-dimensional diffractive optical elements

Appl. Phys. Lett. 93, 031101 (2008); 10.1063/1.2959092

Phase tunable multilevel diffractive optical element based single laser exposure fabrication of three-dimensional photonic crystal templates

Appl. Phys. Lett. 91, 061122 (2007); 10.1063/1.2768867

Electrically tunable two-dimensional holographic photonic crystal fabricated by a single diffractive element Appl. Phys. Lett. 89, 171101 (2006); 10.1063/1.2364471

Design and holographic fabrication of tetragonal and cubic photonic crystals with phase mask: toward the massproduction of three-dimensional photonic crystals

Appl. Phys. Lett. 86, 071117 (2005); 10.1063/1.1865329

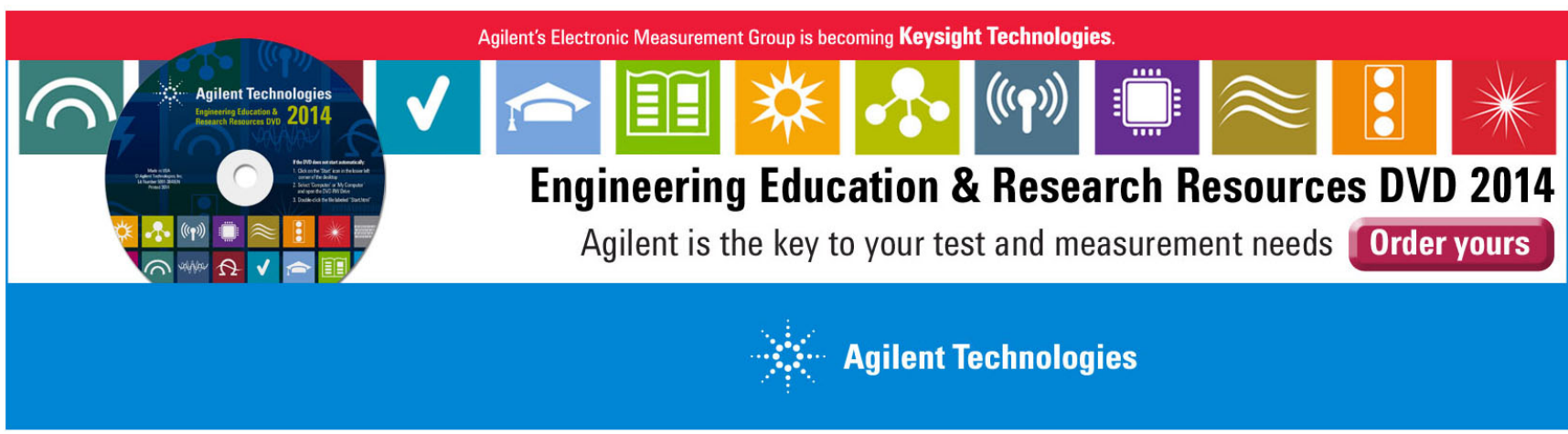




\title{
Holographic fabrication of three-dimensional orthorhombic and tetragonal photonic crystal templates using a diffractive optical element
}

\author{
Zsolt Poole, Di Xu, and Kevin P. Chen ${ }^{\mathrm{a})}$ \\ Department of Electrical and Computer Engineering, University of Pittsburgh, Pittsburgh, Pennsylvania \\ 15261, USA \\ Isai Olvera, Kris Ohlinger, and Yuankun Lin \\ Department of Physics and Geology, University of Texas-Pan American, Edinburg, Texas 78541, USA
}

(Received 6 June 2007; accepted 1 October 2007; published online 17 December 2007)

\begin{abstract}
We report the fabrication of both orthorhombic and tetragonal photonic crystal templates using a phase mask technique. Three-dimensional photonic crystal structures were formed by a double exposure of SU8 to three-beam interference patterns generated by a phase mask. Lattice structures and photonic band gap can be controlled by rotational angles of the phase mask between two exposures. Band gap computation predicts that photonic crystal structures with the optimized band gap can be realized when the rotational angle is set between $50^{\circ}$ and $70^{\circ}$. A photonic crystal template with $60^{\circ}$ phase mask rotation was fabricated, showing improved lattice structures required for the band gap opening. (C) 2007 American Institute of Physics. [DOI: 10.1063/1.2822844]
\end{abstract}

Photonic crystals are artificially engineered periodic microstructures composed of alternating high and low refractive index materials. Since its introduction in 1987, , $^{1,2}$ extensive theoretical and experimental studies have revealed many unique properties of photonic crystals useful in optical communication. ${ }^{3,4}$ Intrigued by their vast potential in photonics engineering, tremendous efforts have been invested into the fabrication of defect-free three-dimensional (3D) photonic crystal structures with complete band gaps around the visible and near infrared telecommunication windows. ${ }^{5-8}$ So far, a number of fabrication techniques such as conventional multilayer stacking of woodpile structures using semiconductor fabrication processes, ${ }^{5}$ colloidal self-assembly, ${ }^{6}$ multiphoton direct laser writing, ${ }^{7}$ and multibeam holographic lithography ${ }^{4,8-14}$ have been employed to produced submicron 3D photonic crystals or templates. With appropriate infiltration and inversion processes, ${ }^{15}$ these $3 \mathrm{D}$ templates can be converted into 3D photonic crystal structures with complete band gaps in the visible and the near infrared regions.

Among all crystal fabrication techniques, multibeam holographic lithography is the most attractive for its controllability and flexibility. Traditionally, multibeam interference holography was realized by a large number of bulk optical components, such as mirrors, beam splitters, and lenses. These optical setups, due to their high degrees of freedom, are among the most difficult to align and are highly susceptible to thermal and mechanic variations. Recently, a number of groups have demonstrated the construction of multibeam interference using a single diffractive or deflective optical element. ${ }^{10-13}$ The holographic lithography based on multiple diffractive elements on one glass mask ${ }^{10}$ and a single flat-top prism $^{11}$ was demonstrated for the fabrication of photonic crystal template. By employing two-layer orthogonally aligned phase masks, photonic crystals with woodpile structures have been recently demonstrated through one or two laser exposures. ${ }^{12-14}$ The utilization of single optical element can effectively reduce complexity of the optical setup and its vulnerability to the thermal and mechanical variations. ${ }^{10,11}$ When the multibeam interference is constructed by bulk op-

${ }^{a)}$ Electronic mail: kchen@engr.pitt.edu tical elements, the interference laser beams can be directed from all spaces $\left(360^{\circ}\right){ }^{4}$ However, the interference pattern generated through a single optical element can only comes from the same half-space, which leads to elongated 3D structures in the $z$ direction. Thus, the corresponding lattice structure has a reduced symmetry and the photonic band gap shrinks.

In this letter, we demonstrate that the elongation in the $z$ direction can be compensated by rotating a phase mask by an appropriate angle between $50^{\circ}$ and $70^{\circ}$, which increases the lattice constant in other direction. The 3D photonic crystal template formed in SU8 can have orthorhombic or tetragonal structure depending on the rotational angle. Theoretical calculation predicts that the optimized rotation angle of a phase mask can achieve up to $50 \%$ increase in photonic band gap compared with those formed by two orthogonally oriented phase masks.

The basic approach utilized to fabricate an interconnected periodic polymeric structure is the double exposure of photosensitive material to three interfering laser beams generated by a one-dimensional phase mask, as shown in Fig. 1(a). A linearly polarized beam from an argon ion laser at $514.5 \mathrm{~nm}$ is expanded, collimated, and passed through a phase mask to produce two first order and one zeroth order diffracted beams (intensity ratio of 1:5). A layer of photoresist on a glass wafer is first exposed to the interference of the three laser beams. Thus, a spatially modulated chemical change in the photoresist is produced. A second rotated and translated phase mask is then used to induce a second set of spatially modulated chemical changes in the photoresist. The orientation of the second interference pattern is controlled by the orientation angle $\alpha$ of the second phase mask with respect to the first [Fig. 1(a)]. To form an interconnected 3D woodpile structure, the phase mask was shifted along the optical axis ( $c$ axis) by a distance $\Delta z$ for the second exposure. This shift has a significant impact on the size of overlap between the two interference patterns and consequently on the size of the band gap formed in the final structure. A translation of $\Delta z=0.25 c$ of the second interference pattern along the $c$ axis yields an optimized fully interconnected woodpile structure, as shown in Fig. 1(b). High-precision 

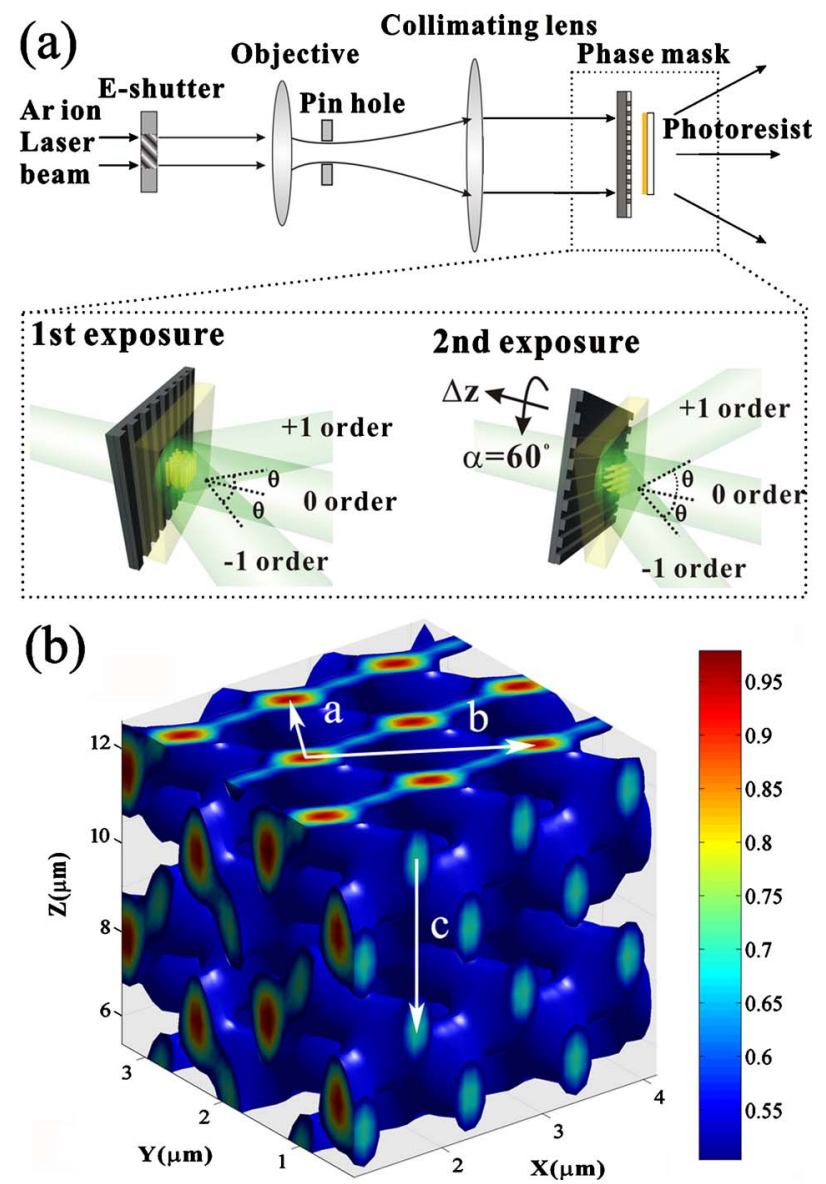

FIG. 1. (Color online) (a) Schematic sketch of the experimental setup. (b) Simulated three-dimensional woodpile structures through double exposures. The rotational angle of phase mask is $60^{\circ}$. The scale bar shows the accumulated laser energy density after two exposures.

motion stages (Newport PM500-4L) were used to control the movements of the phase masks with $\pm 100 \mathrm{~nm}$ accuracy. By controlling the rotational angle and the relative shift of the phase mask along the optic axis, both orthorhombic and tetragonal photonic crystal structures were formed. Figure 1(b) shows a simulated face-centered-orthorhombic photonic crystal structure formed by rotating the phase mask by $\alpha$ $=60^{\circ}$ between two exposures. The lattice constants $(a, b$, and c) labeled in Fig. 1(b) are determined by the angle of diffraction $\theta$ of the first order beams in the photoresist and by the angular rotation of the phase mask $\alpha$ as $L /[\cos (\alpha / 2)]$, $L /[\sin (\alpha / 2)]$, and $L[\cot (\theta / 2)],{ }^{13}$ respectively, where $L$ is the grating period given by $L=\lambda / \sin \theta, \lambda$ is the laser wavelength in the photoresist material.

If a negative tone photoresist (such as SU8) is used to generate the crystal template, an inversion process can turn these structures into high-index contrast (e.g., Si/air) photonic crystals. ${ }^{15}$ Band gap calculation, using MIT PHOTONICBANDS package ${ }^{16}$ reveals the existence of a global band gap, as shown in the inset of Fig. 2. This calculation was performed for the interference pattern generated by a $60^{\circ}$ rotation of the phase mask $\left(\alpha=60^{\circ}\right)$ and a $c / L$ ratio of 2 . The resultant face-centered-orthorhombic structure exhibited a full photonic band gap of $17 \%$ intensity. Figure 2 depicts the significance of the overlap between the two alternating highintensity stacks controlled by the translation $\Delta z$ of the second phase mask along the optical axis. A global band gap of $4 \%$ exists in structures with $\alpha=60^{\circ}$ and $\Delta z=0.03 c$. In structures

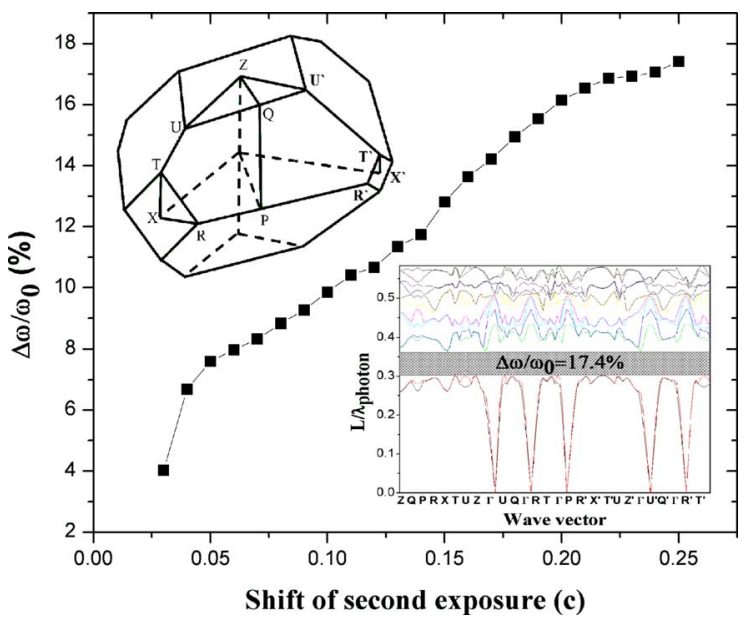

FIG. 2. (Color online) Photonic band gap as a function of the phase mask displacement $\Delta z$ between two exposures. The phase mask rotational angle is $60^{\circ}$. The insets are the first Brillouin surface and photonic band diagram for the face-centered-orthorhombic structure.

where $\Delta z \leqslant 0.03 c$, the width of the band gap reduces rapidly and ultimately vanishes. A maximum band gap of $17 \%$ was achieved at a shift, $\Delta z=0.25 c$.

To study the dependence of the size of the band gap on $\alpha$, photonic band gap calculations were performed with various $c / L$ ratios, as shown in Fig. 3. Since all the laser beams come from the same half-space, the interference pattern generated will be elongated along the $c$ axis due to relatively small interference angles. This elongation, along with a rotational angle of $90^{\circ}$, causes the lattice constant $c$ to be larger than $a$ and $b$, yielding a face-center-tetragonal structure. When the phase mask's rotational angle decreases from $90^{\circ}$, the lattice constant $b$ increases, while $a$ decreases, in effect reducing the photonic crystal structure to a lattice with orthorhombic symmetry. A small phase mask rotational angle $\alpha$ can transfer the lattice back into tetragonal again when the lattice constant $b$ is equal to $c$. When the value of $b$ approaches that of $c$, the structure becomes more symmetric and the band gap increases. From simulation, we found that the maximum band gap occurs when the structure has the highest possible symmetry. For a relatively small $c / L$ ratios, where $c$ approaches $a$ and $b$ and $\alpha=90^{\circ}$, the widest band gap is produced. For larger $c / L$ ratios, the maximum band gap occurs at a rotational angle $\alpha \neq 90^{\circ}$. Figure 3 illustrates the

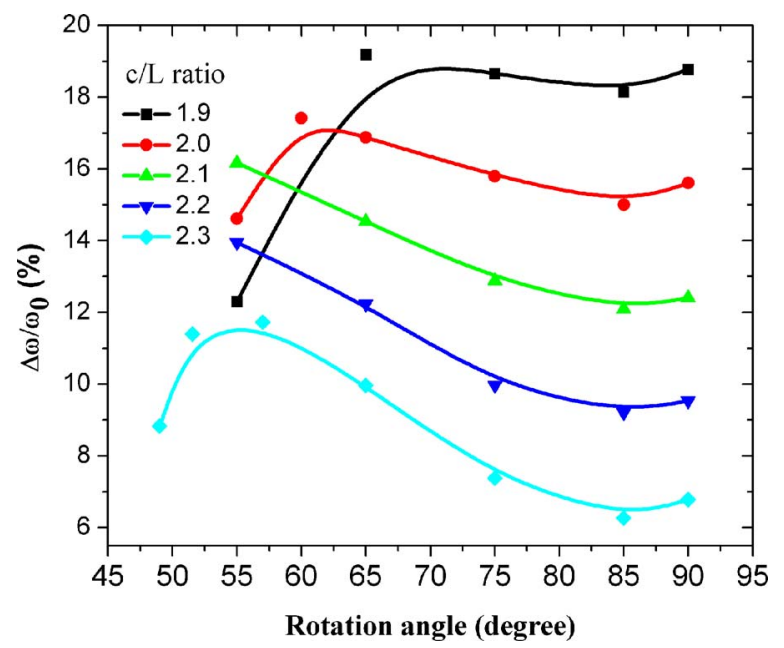

FIG. 3. (Color online) Photonic band gap as a function of the phase mask rotational angle. 
rotation angles $\alpha$ that maximize the band gap for structures with a large $c / L$ values. When $c$ is larger than $1.9 L$, a small rotational angle of the phase mask is required to maximize the band gap. As shown in Fig. 3, for $c / L=2.0$, a $60^{\circ}$ rotational angle maximizes the photonic band gap. Maximizing the band gap for structures with $c / L$ ratios larger than 2 requires less than $60^{\circ}$ angular displacements. For this $c / L$ ratio, varying the rotation angle from $90^{\circ}$ initially results in a drop in the width of the gap followed by an increase. This is consistent with the symmetry transformation of the photonic structure, changing from tetragonal symmetry to orthorhombic symmetry then back to tetragonal symmetry.

To demonstrate the feasibility of the proposed fabrication technique, both orthorhombic and tetragonal structures were recorded into a modified SU8 photoresist (from MicroChem Corp.). To produce orthorhombic structure with $60^{\circ}$ phase mask rotation, the photoresist solution was prepared by mixing $40 \mathrm{~g} \mathrm{SU8-2035}$ with $0.5 \mathrm{wt} \%$ (relative to SU8 2035) of 5,7-diiodo-3-butoxy-6-fluorone (H-Nu470), $2.5 \mathrm{wt} \%$ of (4-octyloxphenyl) phenyl iodonium hexafluoroantimonate (OPPI) and $10 \mathrm{ml}$ propylene carbonate to assist the dissolution. Due to the large background energy presented in the generated interference pattern $(53 \%$ of zeroth order), the photoresist solution was further modified by the addition of $20 \mathrm{~mol} \%$ triethylamine. ${ }^{9}$ Subsequent exposure to light generates Lewis acids that are vital in the cross-linking process during postexposure bake. The addition of triethylamine, acting as an acid scavenger, allowed the formation of an energy gap which prevented the polymerization process in locations exposed below the energy threshold. The substrates utilized for crystal fabrication were polished glass slides cleaned with piranha solution and dehumidified by baking on a hot plate at $200{ }^{\circ} \mathrm{C}$ for $20 \mathrm{~min}$. Each substrate was precoated with $1 \mu \mathrm{m}$ layer of Omnicoat (Microchem) to enhance adhesion. The SU8 mixture was spin coated onto the pretreated substrate at speeds between 700 and $1500 \mathrm{rpm}$, resulting in a range of thicknesses from 25 to $5 \mu \mathrm{m}$. Prebake of SU8 films was preformed at a temperature of $65^{\circ} \mathrm{C}$ for about $30 \mathrm{~min}$. The prepared samples were first exposed under $500 \mathrm{~mW}$ illumination for $0.9 \mathrm{~s}$ using the first phase mask. A second phase mask, which was rotated by $\alpha$ about the optic axis and translated by $\Delta z$ with respect to the first, was then used for an additional $0.9 \mathrm{~s}$ exposure. The samples were postbaked at $65{ }^{\circ} \mathrm{C}$ for $10 \mathrm{~min}$ and $95{ }^{\circ} \mathrm{C}$ for $5 \mathrm{~min}$ and immersed in SU8 developer (Microchem) for $5 \mathrm{~min}$.

Figure 4(a) shows a scanning electron microscopy (SEM) top view picture of a woodpile orthorhombic structure recorded in SU8 with an $\alpha$ of $60^{\circ}$. The inset of the same figure details the predicted structure from simulation. The three-dimensional span of the structure visible in Fig. 4(b) was also imaged by a SEM. The layer-by-layer woodpile nature of the structure is clearly demonstrated. The overlapping and cross connection of neighboring layers ensure a stable formation of three-dimensional structures for further processing. From Figs. 4(a) and 4(b), we measured by SEM that the lattice constant $b=1.3 \mu \mathrm{m}$ and $c=3.4 \mu \mathrm{m}$. The elongation in the $z$ direction was thus compensated by the $60^{\circ}$ rotation, compared with $b=1.06 \mu \mathrm{m}$ and $c=6.13 \mu \mathrm{m}$ in the structure generated by two orthogonally oriented phase masks with similar period used in this work. ${ }^{14}$

In summary, we demonstrate the fabrication of threedimensional photonic crystal templates in SU8 using phase mask bassed hoiographic lithography. The stîticture and sym-
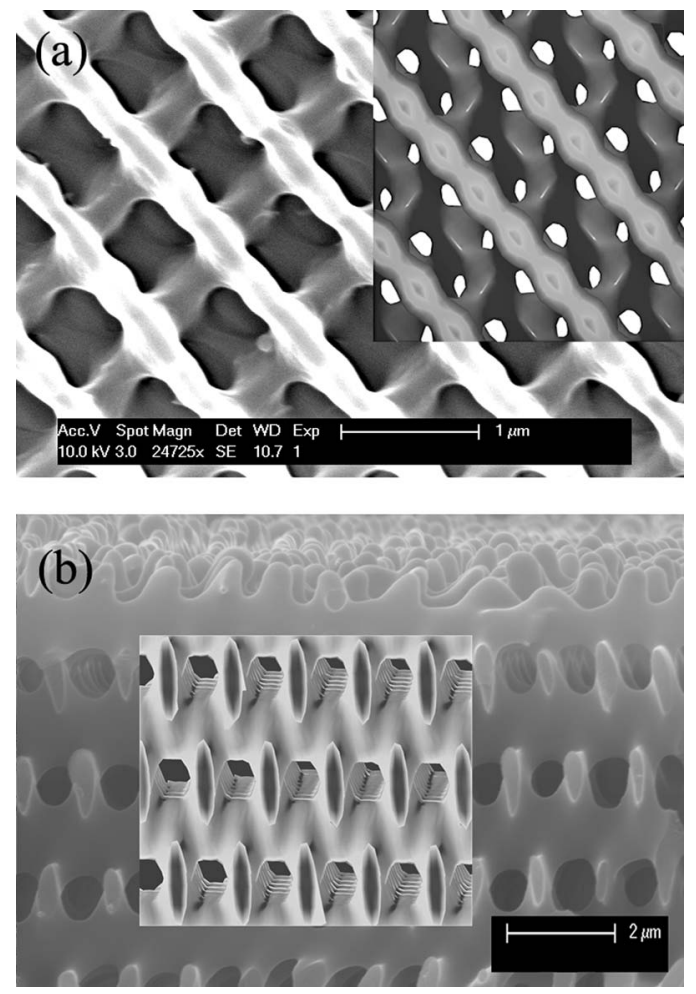

FIG. 4. (a) A SEM top view picture and (b) a SEM side view picture of a woodpile orthorhombic structure recorded in SU8 with an $\alpha$ of $60^{\circ}$. Simulated structures are inserted in figures.

metry of the photonic crystals formed can be controlled by the rotational angle of a phase mask to compensate the structural elongation in the $z$ direction and to enlarge the photonic band gap. Photonic band gap computations predict the existence of optimum band gaps in those woodpile structures with $\alpha$ between $50^{\circ}$ and $70^{\circ}$ instead of traditional $90^{\circ}$ rotation.

This work is supported by National Science Foundation under Award Nos. CMMI-0609345 (Y.L.) and CMMI0637065 (K.P.C.).

${ }^{1}$ S. John, Phys. Rev. Lett. 58, 2486 (1987).

${ }^{2}$ E. Yablonovitch, Phys. Rev. Lett. 58, 2059 (1987).

${ }^{3}$ M. Maldovan and E. L. Thomas, Nat. Mater. 3, 593 (2004).

${ }^{4}$ O. Toader, T. Y. M. Chan, and S. John, Phys. Rev. Lett. 92, 043905 (2004).

${ }^{5}$ K. M. Ho, C. T. Chan, C. M. Soukoulis, R. Biswas, and M. Sigalas, Solid State Commun. 89, 413 (1994).

${ }^{6}$ A. Hynninen, J. H. J. Thijssen, E. C. M. Vermolen, M. Dijkstra, and A. V. Blaaderen, Nat. Mater. 6, 202 (2007).

${ }^{7}$ M. Deubel, G. V. Freymann, M. Wegener, S. Pereira, K. Busch, and C. M. Soukoulis, Nat. Mater. 3, 444 (2004).

${ }^{8}$ M. Campbell, D. N. Sharp, M. T. Harrison, R. G. Denning, and A. J. Turberfield, Nature (London) 404, 53 (2000).

${ }^{9}$ S. Yang, M. Megens, J. Aizenberg, P. Wiltzius, P. M. Chaikin, and W. B. Russel, Chem. Mater. 14, 2831 (2002).

${ }^{10}$ I. Diviliansky, T. S. Mayer, K. S. Holliday, and V. H. Crespi, Appl. Phys. Lett. 82, 1667 (2003).

${ }^{11}$ Y. V. Miklyaev, D. C. Meisel, A. Blanco, G. V. Freymann, K. Busch, W. Koch, C. Enkrich, M. Deubel, and M. Wegener, Appl. Phys. Lett. 82, 1284 (2003).

${ }^{12}$ Y. Lin, P. R. Herman, and K. Darmawikarta, Appl. Phys. Lett. 86, 071117 (2005).

${ }^{13}$ Y. Lin, D. Rivera, and K. P. Chen, Opt. Express 14, 887 (2006).

${ }^{14}$ D. Chanda and P. R. Herman, Appl. Phys. Lett. 91, 061122 (2007).

${ }^{15}$ N. Tétreault, G. V. Freymann, M. Deubel, M. Hermatschweiler, F. P. Willard, S. John, M. Wegener, and G. A. Ozin, Adv. Mater. (Weinheim, Ger.) 18, 457 (2006).

${ }^{6}$ S. G. Johnson and J. D. Joannopouios, Opt. Express 8, 173 (2001). 